\title{
The Effect of Extracorporeal Albumin Dialysis (ECAD) on Pruritus Using MARS Versus New Adsorbent Recirculation (OPAL)
}

ISSN: 2637-7632

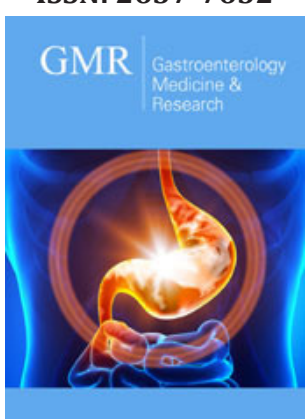

*Corresponding author: Jan Stange, Department Internal Medicine, University of Rostock, Germany

Submission: 倫July 16, 2020

Published: 制July 27, 2020

Volume 4 - Issue 5

How to cite this article: Hartmut Schmidt, Christian Wilms, Hans Ulrich Gerth, Jan Stange, et al. The Effect of Extracorporeal Albumin Dialysis (ECAD) on Pruritus Using MARS Versus New Adsorbent Recirculation (OPAL). Gastro Med Res. 4(5). GMR.000600. 2020

DOI: 10.31031/GMR.2020.04.000600

Copyright@ Jan Stange, This article is distributed under the terms of the Creative Commons Attribution 4.0 International License, which permits unrestricted use and redistribution provided that the original author and source are credited.
Hartmut Schmidt ${ }^{1}$, Christian Wilms ${ }^{1}$, Hans Ulrich Gerth ${ }^{1}$, Michael Hinz ${ }^{2}$, Sebastian Koball ${ }^{2}$, Alexandra Rivera Luna ${ }^{2}$, Victoria Rivera Luna ${ }^{2}$, Claudia Pfensig $^{2}$, Adrian Dominik ${ }^{2}$, Andreas Kortgen ${ }^{3}$, Christoph Sponholz ${ }^{3}$, Matthias Dollinger ${ }^{4}$, Sebastian Klammt ${ }^{2}$, Kristin Bruederlein ${ }^{2}$, Steffen Mitzner ${ }^{2}$, Raoul Benedilkt Sauer ${ }^{2}$ and Jan Stange ${ }^{2 *}$

${ }^{1}$ Department Internal Medicine, University of Muenster, Germany

${ }^{2}$ Department Internal Medicine, University of Rostock, Germany

${ }^{3}$ Department Intensive Care and Anesthesiology, University of Jena, Germany

${ }^{4}$ Department Internal Medicine University of Ulm, Germany

\begin{abstract}
Extracorporeal Albumin Dialysis (ECAD) is a therapy option for pruritus in cholestatic liver disease. The aim was to evaluate the feasibility for ECAD using two different devices. A subgroup with intractable pruritus of a prospective randomized crossover study in liver patients was analyzed. Randomly subjects received either MARS ${ }^{\mathrm{TM}}$ first and new HepalbinTMCluster12 albumin dialysis (OPAL ${ }^{\mathrm{TM}}$ ) second or vice versa. Visual Analogue Score, total bile acids as well as albumin binding function were measured before and after each treatment. 8 subjects suffering from cholestatic liver disease and pruritus were enrolled. Subjects in both arms were comparable. Albumin dialysis could reduce albumin bound toxins, the binding function of patient's albumin improved in OPAL, but not in MARS. Which one of the biomarker changes are responsible for the improvement of pruritus remains unknown and requires more research. The procedure was safe in all subjects.
\end{abstract}

\section{Introduction}

Extracorporeal albumin dialysis (ECAD) is a therapy where the dialysis solution is enriched with human albumin in order to enhance the removal of albumin bound toxins (ABT), which are thought to play a key role in cholestatic pruritus and other liver decompensations [1]. ECAD has been shown to be effective and safe in case reports and small clinical trials outside the United States in relieving medically refractory pruritus in a variety of cholestatic conditions [2]. In order to maintain an effective concentration gradient for ABT, effective ECAD requires the use of highly purified albumin in the dialysate. Recently, four experienced liver centers could show in a prospective randomized cross-over study (EUDAMED: CIV-13-04010642) that implementing a new adsorbent (HepalbinTMCluster12) for dialysate albumin regeneration in the OPAL ${ }^{\mathrm{TM}}$ system resulted into a new quality of ECAD by not only removing ABTs more effectively, but the higher detoxification dose delivered also led to an improvement of patient's endogenous albumin binding function [3] measured by Electron Spin Resonance Spectroscopy (ESR). Although MARS ${ }^{\mathrm{TM}}$ has been shown in the past to remove ABTs it had failed in a past study to improve ESR, which is considered a potential surrogate for survival [4]. How the performance of the new adsorbent affects effectiveness of pruritus treatment has not been shown in a larger cohort yet.

\section{Aim}

The aim of this sub study of [3] is to demonstrate the feasibility of the new adsorbent technology to address intractable cholestatic pruritus which was one out of three indications 
for ECAD to enter the study and measure biomarkers correlated with pruritus as well as the albumin binding function for such biomarkers.

\section{Patients and Methods}

Out of 30 patients screened and enrolled in the OPALESCE study [3], 8 presented with pruritus as an indication. Patients were randomized in the study to receive MARS ${ }^{\mathrm{TM}}$ on day 1 and $\mathrm{OPAL}^{\mathrm{TM}}$ on day 2 or vice versa. The Visual Analogue Scale (VAS) for Pruritus, Bile Acids and Albumin Binding Function using ESR were measured before and after each treatment on day 1 and 2. All OPAL ${ }^{\mathrm{TM}}$ and all MARS $^{\mathrm{TM}}$ treatments were pooled.

\section{Results}

Out of 8 patients 4 had cholestatic liver disease, 3 drug induced cholestasis and one had secondary sclerosing cholestasis with pruritus and had failed standard of care. There were no significant differences between patients initiating with MARS ${ }^{\mathrm{TM}}$ and those with OPAL ${ }^{\mathrm{TM}}$ at baseline with respect to MELD, CTP, or VAS scores. All subject had one evaluable MARS ${ }^{\mathrm{TM}}$ and one evaluable OPAL ${ }^{\mathrm{TM}}$ treatment, so there were 8 of each with pre/post pruritus VAS assessment and laboratory values. As observed in studies before, both methods of ECAD resulted into a significant reduction of total bile acids (Wilcoxon signed rank test, $\mathrm{p}<0.01$ ) which was associated with a reduction of pruritus in $5\left(\mathrm{MARS}^{\mathrm{TM}}\right)$ and $6\left(\mathrm{OPAL}^{\mathrm{TM}}\right)$ patients during a single treatment of 6 hours (no statistics applied), see Figure 1 . The therapy was safe, no severe adverse events related to the therapy were reported. The ESR detection of improvement of patient's albumin binding function for spin labelled lipids (16 doxyl stearate), expressed as patient's albumin binding efficiency and detoxification efficacy [4] is shown in Figure 2. As observed before, in this cohort a single MARSTM therapy did not significantly improve ESR, although ECAD using the HepalbinTM adsorbents with a higher capacity for fatty acids resulted in a significant increase of both ESR parameters, as published before for the complete study [3].
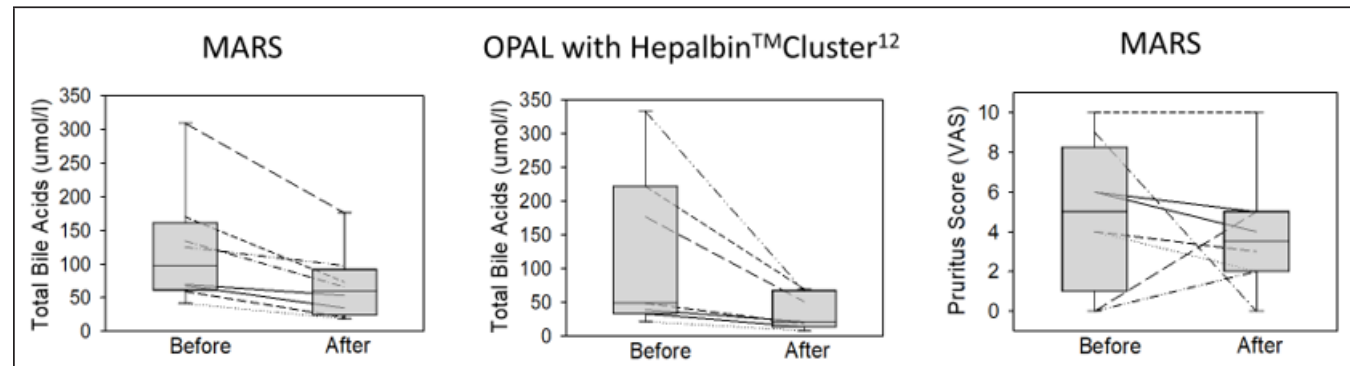

OPAL with Hepalbin ${ }^{\text {TM}}$ Cluster $^{12}$

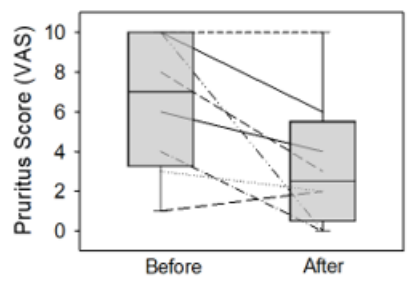

Figure 1: The total bile acids before and after each MARS and each OPAL treatment and pre/post bile acid levels displayed as a box plot for the median, $10^{\text {th }}, 25^{\text {th }}, 75^{\text {th }}$ and $90^{\text {th }}$ percentile (left graphs). On the right is the individual VAS pruritus score for each patient and the pre/post VAS displayed as a box plot for the median, $10^{\text {th }}, 25^{\text {th }}, 75^{\text {th }}$ and $90^{\text {th }}$ percentile.

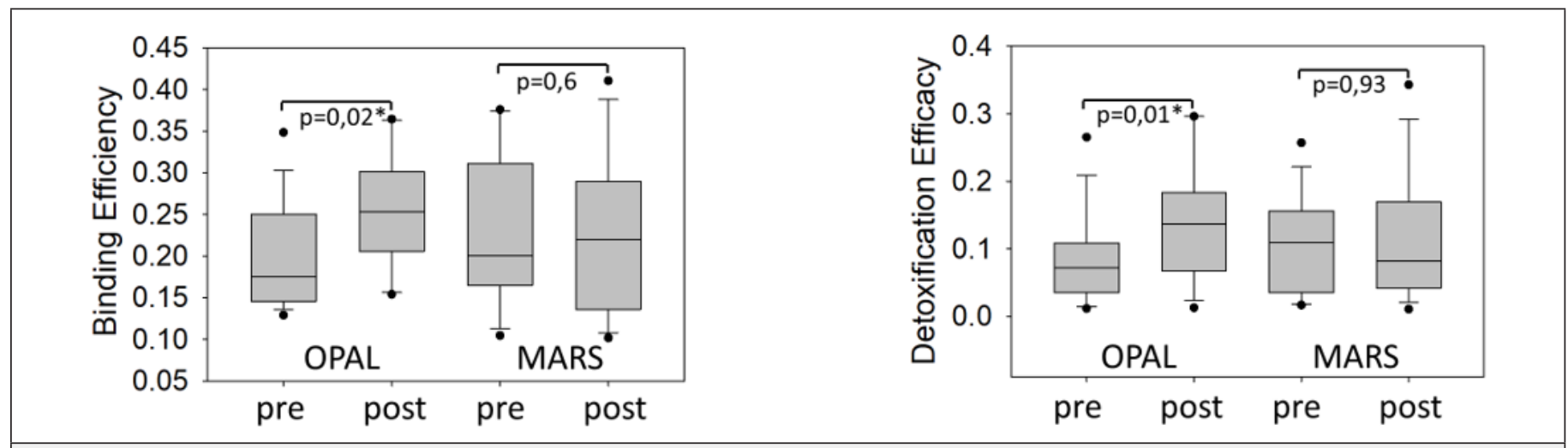

Figure 2: Binding Efficiency (left) and Detoxification Efficacy (right) measured by ESR before and after OPAL and MARS. OPAL, but not MARS significantly improved during a single 6 hour therapy.

\section{Discussion}

An effect of OPAL ${ }^{\mathrm{TM}}$ on pruritus and bile acids as reported for ECAD repeatedly before could be confirmed. Since recent hypothesis points at a potential role for autotoxin mediated metabolism of phospholipids in pruritus pathogenesis albumin binding function for phospholipids may play a role in the mechanism of action for
ECAD in pruritus. Recently, the group filed for an Investigational Device Exemption to conduct a trial to confirm this hypothesis.

\section{References}

1. Stange J (2011) Extracorporeal liver support. Organogenesis 7(1): 6473. 
2. Leckie P, Tritto G, Mookerjee R, Davies N, Jones D, et al. (2012) Outpatient albumin dialysis for cholestatic patients with intractable pruritus. Aliment Pharmacol Ther 35(6): 696-704.

3. Kortgen A, Stange J, Sponholz C, Schmidt H, Wilms C, et al. (2018) Opal, but not MARS improves patient's albumin binding function measured by Electron Spin Resonance (ESR) in a prospective multicenter trial. Journal of Hepatology 68: 365-604
4. Jalan R, Schnurr K, Mookerjee RP, Sen S, Cheshire L, et al. (2009) Alterations in the functional capacity of albumin in patients with decompensated cirrhosis is associated with increased mortality. Hepatology 50(2): 555-564. 